\title{
Internet security applications of the Munn rings
}

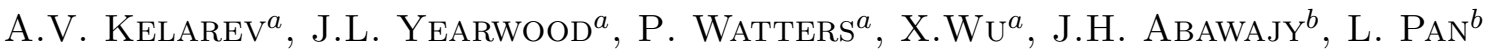 \\ ${ }^{a}$ Internet Commerce Security Laboratory \\ Centre for Informatics and Applied Optimization \\ Graduate School of Information Technology and Mathematical Sciences \\ University of Ballarat, P.O. Box 663, Ballarat, Victoria 3353, Australia \\ $\{$ a.kelarev, j. yearwood, p. watters, x. wu $@$ ballarat.edu.au \\ ${ }^{b}$ School of Information Technology, Deakin University, \\ 221 Burwood Highway, Melbourne, Victoria 3125, Australia \\ \{jemal.abawajy,l.pan\}@deakin.edu.au \\ Communicated by M. V. Volkov \\ Dedicated to the memory of Walter Douglas Munn
}

\begin{abstract}
Effective multiple clustering systems, or clusterers, have important applications in information security. The aim of the present article is to introduce a new method of designing multiple clusterers based on the Munn rings and describe a class of optimal clusterers which can be obtained in this construction.
\end{abstract}

\section{Introduction}

Douglas Munn has obtained many fundamental results on various ring constructions, see, for example, the monograph [16] and papers [9, 10,29-37]. The concept of a Munn ring plays crucial roles in the investigation of several other ring constructions and has been used by many researchers. To illustrate, let us refer to the most recent paper [1] dealing with an application of the Munn rings in representation theory. Often Munn rings are used implicitly in the proofs, as for example, in [12], see also [40]. Section 3.12 of the book [16] is devoted to the Munn rings, see also Sections 4.3 and 4.5.

Let $R$ be a ring, $I$ and $\Lambda$ nonempty sets, and let $P$ be a $\Lambda \times I$-matrix with entries in $R$. A Munn ring over $R$ with sandwich matrix $P$ is the set $M(R ; I, \Lambda ; P)$, consisting of all $I \times \Lambda$ matrices with a finite number of nonzero entries over $R$, equipped with the usual addition and multiplication $\cdot$ defined by $A \cdot B=A P B$, for $A, B \in M(R ; I, \Lambda ; P)$. Originally, this construction was motivated by analogy with completely 0 -simple semigroups, see $[14,39]$ for examples of recent results.

The aim of the present paper is to introduce a new method of designing multiple clustering systems, or clusterers, based on the Munn rings and describe a class of optimal clusterers which can be obtained in this construction.

The design of multiple clustering systems has important applications in information security (see, for example, [6], [11], [28] and [38]). Several individual clusterers are often developed for the analysis 
of malware, and then it is a challenging task to combine them into one multiple clusterer capable of correcting possible errors of the individual clusterers.

Clustering and classification of data play central roles in practical applications of data mining technologies, including information security. This is a novel area where full potential for the application of semigroup and ring constructions has not been explored yet. For broad background information we refer the readers to the monographs [41,45] and papers [2-5, 15, 19, 21-23, 25, 43, 44] and include only a very brief overview here.

Classification deals with known classes of data. These classes are represented by given samples of data. The samples are used for supervised training of the classifier to enable it to recognize new elements of the same known classes. On the other hand, clustering handles data without known sensible groupings or clusters. The task of a clustering system, or clusterer, is to conduct unsupervised investigation of the data in order to determine new groupings or clusters.

\section{Prerequisites}

This section contains only a concise review of prerequisites on clusterers and their cluster sets required for our theorems. We use standard concepts and refer the readers to $[5,13,16-18,41,45]$ for general background information. For more details on related constructions, we refer to $[7,8,20,26,27]$.

Let $p$ be a prime, $q$ a power of $p$, and let $F=F_{q}=G F(q)$ be the finite field of order $q$. Throughout $I$ and $\Lambda$ are finite nonempty sets and $R=M(F ; I, \Lambda ; P)$ is a Munn ring over $F$ with sandwich-matrix $P$.

For $i \in I, \lambda \in \Lambda$, denote by $e_{i \lambda}$ the standard elementary matrix with 1 in the intersection of $i$-th row and $\lambda$-th column and zeros in all other entries. Clearly, every element $r \in R$ has a unique representation of the form $r=\sum_{i \in I, \lambda \in \Lambda} r_{i \lambda} e_{i \lambda}$, where all $r_{i \lambda} \in F$.

Further, we assume that the number $m$ of individual clusterers being combined is equal to the dimension $m=|I| \times|\Lambda|$ of $R$. Since $R$ has dimension $m$ over $F$, we can follow the general approach initiated in [24] and endow $F^{m}$ with a multiplication by identifying $F^{m}$ with $R$. This allows us to consider the cluster sets of multiple clusterers as subsets of $R$ and use small numbers of generators to define multiple clusterers.

The weight $\operatorname{wt}(r)$ of $r=\sum_{i \in I, \lambda \in \Lambda} r_{i \lambda} s_{i \lambda} \in R$ is the number of nonzero coefficients $r_{i \lambda}$ in $r$. The weight of a subset $C \subseteq F^{m}$ is the minimal weight of a nonzero element in $C$. The minimum distance of $C$ is the smallest weight of a nonzero difference $u-v$ for $u, v \in C$. If $C$ is a linear space, then the minimum distance of $C$ coincides with its weight. The information rate of a cluster set $C$ in $F^{m}$ can be defined as $\log _{q}(|C|) / m$.

We investigate cluster sets which can be defined as ideals in $R$. An ideal generated by the elements $g_{1}, \ldots, g_{k} \in R^{1}$ is the set

$$
\operatorname{id}\left(g_{1}, \ldots, g_{k}\right)=R^{1} g_{1} R^{1}+\cdots+R^{1} g_{k} R^{1}=\left\{\sum_{j=1}^{m_{1}} \ell_{1, j} g_{1} r_{1, j}+\cdots+\sum_{j=1}^{m_{k}} \ell_{k, j} g_{k} r_{k, j} \mid \ell_{i, j}, r_{i, j}, \in R^{1}\right\},
$$

where $R^{1}=R \oplus F_{q}$ stands for the ring $R$ with identity 1 adjoined in a standard fashion. 
According to [41], Section 7.5, for a clusterer with a cluster set $C$ to be efficient, the set $C$ must satisfy the following properties:

(A1) The weight of $C$ must be large.

(A2) The information rate of $C$ must be large.

(A3) A convenient method of generating the set $C$ is essential.

(A4) If all vectors of $C$ are recorded in a matrix $M$, then there should not be strong correlation or functional dependencies between small sets of columns of $M$. In particular, the matrix $M$ should not have duplicate columns.

Additional properties may be required depending on the practical application being considered.

\section{Main Results}

For the sandwich-matrix $P$, let us define subsets

$$
\begin{aligned}
& J=\left\{j \in I \mid p_{\mu j}=0(\forall \mu \in \Lambda)\right\}, \\
& L=\left\{\mu \in \Lambda \mid p_{\mu j}=0(\forall j \in I)\right\} .
\end{aligned}
$$

The main theorem of this paper determines the largest weights of the cluster sets in the Munn rings, as required in (A1).

Theorem 1 Let $R=M(F ; I, \Lambda ; P)$ be a Munn ring over $F$ such that each row and every column of $P$ has at most one nonzero entry. Then the following conditions hold:

(i) the largest weight of a cluster set of the form $\operatorname{id}\left(g_{1}, \ldots, g_{k}\right)$ in $R$ is equal to

$$
W=\max \{|J|,|L|,|J| \times|L|\} ;
$$

(ii) the maximum number of errors of individual clusterers, which can be corrected by a multiple clusterer of the form $\operatorname{id}\left(g_{1}, \ldots, g_{k}\right)$ in $R$ is equal to

$$
E=\max \left\{\left\lfloor\frac{|J|-1}{2}\right\rfloor,\left\lfloor\frac{|L|-1}{2}\right\rfloor,\left\lfloor\frac{|J| \times|L|-1}{2}\right\rfloor\right\} .
$$

Let us now define special generators which occur in all cluster sets of the largest possible weight. Denote by $F^{*}$ the set of all nonzero elements of the field $F$, and put

$$
\begin{aligned}
\mathcal{G}_{J} & =\left\{\sum_{i \in J} r_{i} e_{i \lambda} \mid \lambda \in \Lambda \backslash L, r_{i} \in F^{*}\right\}, \\
\mathcal{G}_{L} & =\left\{\sum_{\lambda \in L} r_{\lambda} e_{i \lambda} \mid i \in I \backslash J, r_{\lambda} \in F^{*}\right\}, \\
\mathcal{G}_{J L} & =\left\{\sum_{i \in J, \lambda \in L} r_{i \lambda} e_{i \lambda} \mid r_{i \lambda} \in F^{*}\right\} .
\end{aligned}
$$


Theorem 2 Let $R=M(F ; I, \Lambda ; P)$ be a Munn ring over $F$ such that each row and every column of $P$ has at most one nonzero entry, and let $C$ be a cluster set of the form $\operatorname{id}\left(g_{1}, \ldots, g_{k}\right)$ with the largest weight in $R$. If $\operatorname{wt}(C)>1$, then $C$ contains an element which belongs to the union of the sets $\mathcal{G}_{J}, \mathcal{G}_{L}$ and $\mathcal{G}_{J L}$.

Proofs of our main theorems follow from the next technical proposition, which describes the ideals generated by the elements of the sets $\mathcal{G}_{J}, \mathcal{G}_{L}$ and $\mathcal{G}_{J L}$.

Proposition 3 Let $R=M(F ; I, \Lambda ; P)$ be a Munn ring over $F$. Then the following conditions hold:

(i) $\operatorname{id}(g)=\left\{\sum_{\mu \in \Lambda} \sum_{i \in J} r_{\mu} r_{i} e_{i \mu} \mid r_{\mu} \in F\right\}$ for every $g=\sum_{i \in J} r_{i} e_{i \lambda} \in \mathcal{G}_{J}, \lambda \in \Lambda$;

(ii) $\operatorname{id}(g)=\left\{\sum_{j \in I} \sum_{\lambda \in L} r_{j} r_{\lambda} e_{i \lambda} \mid r_{j} \in F\right\}$ for every $g=\sum_{\lambda \in L} r_{\lambda} e_{i \lambda} \in \mathcal{G}_{L}, i \in I$;

(iii) $\operatorname{id}(g)=F g$ for every $g \in \mathcal{G}_{J L}$;

(iv) $\operatorname{wt}(\operatorname{id}(g))=\operatorname{wt}(g)=|J|$ for every $g \in \mathcal{G}_{J}$;

(v) $\operatorname{wt}(\operatorname{id}(g))=\operatorname{wt}(g)=|L|$ for every $g \in \mathcal{G}_{L}$;

(vi) $\operatorname{wt}(\operatorname{id}(g))=\operatorname{wt}(g)=|J| \times|L|$ for every $g \in \mathcal{G}_{J L}$.

\section{Proofs}

The annihilator (right annihilator; left annihilator) of $R$ are the sets $\operatorname{Ann}(R)$ (respectively, $\operatorname{Ann}_{r}(R)$; $\left.\operatorname{Ann}_{\ell}(R)\right)$, defined by

$$
\begin{aligned}
\operatorname{Ann}_{r}(R) & =\{x \in R] \mid R x=0\}, \\
\operatorname{Ann}_{\ell}(R) & =\{x \in R \mid x R=0\}, \\
\operatorname{Ann}(R) & =\operatorname{Ann}_{\ell}(R) \cap \operatorname{Ann}_{r}(R) .
\end{aligned}
$$

For any $i, \lambda \in I$, we use the following notation

$$
\begin{aligned}
R_{i \lambda} & =F e_{i \lambda}, \\
S_{i *} & =\sum_{\mu \in \Lambda} F e_{i \mu}, \\
S_{* \lambda} & =\sum_{j \in I} F e_{j \lambda} .
\end{aligned}
$$

If $X \subseteq I, Y \subseteq \Lambda$, then we put

$$
\begin{aligned}
S_{X *} & =\sum_{j \in X} R_{j *}, \\
S_{* Y} & =\sum_{\mu \in Y} R_{* Y}, \\
R_{X Y} & =\sum_{j \in X, \mu \in Y} R_{j \mu} .
\end{aligned}
$$


Lemma 4 Let $F$ be a finite field, and let $R=M(F ; I, \Lambda ; P)$ be a Munn ring over $F$. Then the following equalities hold:

(i) $\operatorname{Ann}_{r}(R)=R_{J *}$;

(ii) $\operatorname{Ann}_{\ell}(R)=R_{* L}$;

(iii) $\operatorname{Ann}(R)=R_{J L}$.

Proof is straightforward and we omit it.

Lemma 4 immediately gives us the following

Lemma 5 Let $F$ be a finite field, and let $R=M(F ; I, \Lambda ; P)$ be a Munn ring over $F$. Then the following inclusions hold:

(i) $\mathcal{G}_{J} \subseteq \operatorname{Ann}_{r}(R)$;

(ii) $\mathcal{G}_{L} \subseteq \operatorname{Ann}_{\ell}(R)$;

(iii) $\mathcal{G}_{J L} \subseteq \operatorname{Ann}(R)$

Proof of Proposition 3. (i): Take any element $g$ in $\mathcal{G}_{J}$. By (2) there exists $\lambda \in \Lambda \backslash L$, and $r_{i} \in F^{*}$, $i \in J$, such that

$$
g=\sum_{i \in J} r_{i} e_{i \lambda}
$$

First, we claim that the set $\mathcal{G}_{J}$ is closed under multiplication by the elements of the form $r e_{j \mu}$, for $j \in I, \mu \in \Lambda, r \in F$. Indeed, $r e_{j \mu} g=0$ by Lemma 5(i). On the other hand,

$$
\operatorname{gre}_{j \mu}=\sum_{i \in J} r r_{i} e_{i \lambda} \cdot e_{j \mu}=\sum_{i \in J}\left(r r_{i} p_{\lambda j}\right) e_{i \mu}
$$

also belongs to $\mathcal{G}_{J}$ since the same element $p_{\lambda j}$ occurs in all of these summands. Therefore

$$
\operatorname{id}(g) \subseteq\left\{\sum_{\mu \in \Lambda} \sum_{i \in J} r_{\mu} r_{i} e_{i \mu} \mid r_{\mu} \in F\right\}
$$

To prove the reverse inclusion, note that by Lemma $5(\mathrm{i})$ expression $(1)$ for $\operatorname{id}(g) \operatorname{simplifies}$ to $\operatorname{id}(g)=$ $g R^{1}$. Since $\lambda \notin L$, there exists $j \in I$ such that $p_{\lambda j} \neq 0$. Hence, for any $\mu \in \Lambda$ and $r_{\mu} \in F$, we get

$$
g \cdot r_{\mu} e_{j \mu}=\sum_{i \in J} r_{\mu} p_{\lambda j} r_{i} e_{i \mu}
$$

in $R$. Therefore condition (i) follows. 
(ii): Take any element $g$ in $\mathcal{G}_{L}$. By (3) there exists $i \in I \backslash J$ and $r_{\lambda} \in F^{*}, \lambda \in L$, such that

$$
g=\sum_{\lambda \in L} r_{\lambda} e_{i \lambda}
$$

It is straightforward to verify that the set $\mathcal{G}_{L}$ is closed under multiplication by the elements of the form $r e_{j \mu}$, for $j \in I, \mu \in \Lambda$, and therefore

$$
\operatorname{id}(g) \subseteq\left\{\sum_{j \in I} \sum_{\lambda \in L} r_{j} r_{\lambda} e_{i \lambda} \mid r_{j} \in F\right\} .
$$

Lemma 5(ii) implies that expression (1) for $\operatorname{id}(g) \operatorname{simplifies~to~} \operatorname{id}(g)=R^{1} g$. Since $i \notin J$, there exists $\mu \in \Lambda$ such that $p_{\mu i} \neq 0$. Therefore, for any $i \in I$ and $r_{i} \in F$, we get

$$
r_{i} e_{j \mu} \cdot g=\sum_{\lambda \in L} r_{i} p_{\mu i} r_{\lambda} e_{j \lambda}
$$

in $R$. Hence condition (ii) follows.

(iii): Take any element $g$ in $\mathcal{G}_{J L}$. By (2) there exist $r_{i \lambda} \in F^{*}, i \in J, \lambda \in L$, such that

$$
g=\sum_{i \in J, \lambda \in L} r_{i \lambda} e_{i \lambda} .
$$

Hence Lemma 5(iii) implies that the expression (1) for id $(g) \operatorname{simplifies~to~} \mathrm{id}(g)=F g$, as required in (iii).

(iv): For every $g=\sum_{i \in J} r_{i} e_{i \lambda} \in \mathcal{G}_{J}$ and $\lambda \in \Lambda$, it is easily seen that the weight of the set

$$
\left\{\sum_{\mu \in \Lambda} \sum_{i \in J} r_{\mu} r_{i} e_{i \mu} \mid r_{\mu} \in F\right\}
$$

is equal to $|J|$. Therefore condition (iv) follows from (i).

(v): For every $g=\sum_{\lambda \in L} r_{\lambda} e_{i \lambda} \in \mathcal{G}_{L}$ and $i \in I$ it is clear that the weight of the set

$$
\left\{\sum_{j \in I} \sum_{\lambda \in L} r_{j} r_{\lambda} e_{i \lambda} \mid r_{j} \in F\right\}
$$

is equal to $|L|$. Therefore condition (v) follows from (ii).

(vi): For every $g \in \mathcal{G}_{J L}$, it is obvious that $\operatorname{wt}(F g)=|J| \times|L|$. Therefore condition (vi) follows from (iii).

Proof of Theorem 2. Let $C=\operatorname{id}\left(g_{1}, \ldots, g_{k}\right)$ be a cluster set with the largest weight in $R$. Suppose that $\operatorname{wt}(C)>1$. Consider several possible cases.

Case 1: $C \subseteq R_{J L}$. Pick a nonzero element $g$ of minimal weight in $C$. Then $g$ has a unique representation of the form

$$
g=\sum_{i \in J, \lambda \in L} r_{i \lambda} e_{i \lambda}
$$


for some $r_{i \lambda} \in F$. Condition (iii) and the maximality of $\operatorname{wt}(C)$ show that $r_{i \lambda} \neq 0$, for all $i \in J, \lambda \in L$. Therefore $g \in \mathcal{G}_{J L}$ in this case.

Case 2: $C \nsubseteq R_{J L}$ and $C \subseteq R_{J *}$. Then there exists an element $r \in C \backslash R_{J L}$. Since $C \subseteq R_{J *}$, we can write

$$
r=\sum_{i \in J, \lambda \in \Lambda} r_{i \lambda} e_{i \lambda}
$$

where all $r_{i \lambda} \in F$. By the choice of $r$, there exists $\mu \notin L$ and $j_{1} \in J$ such that $r_{j_{1} \mu} \neq 0$. The definition of $L$ shows that $p_{\mu j} \neq 0$ for some $j$.

Consider the product $r \cdot e_{j \mu} \in C$. Since every column of $P$ has at most one nonzero entry, we get $p_{\lambda j}=0$ for all $\lambda \neq \mu$. Hence

$$
r \cdot e_{j \mu}=\sum_{i \in J} r_{i \lambda} p_{\mu j} e_{i \mu} \in C .
$$

Since $\operatorname{wt}(C) \leq \operatorname{wt}\left(r \cdot e_{j \mu}\right)$, Proposition 3(i) and the maximality of the weight of $C$ show that $\operatorname{wt}\left(r \cdot e_{j \mu}\right)=$ $|J|$. Therefore $r_{i \lambda} p_{\mu j} \in F^{*}$ for all $i \in J$. This means that $r \cdot e_{j \mu} \in \mathcal{G}_{J}$, and we are done.

Case 3: $C \nsubseteq R_{J L}$ and $C \subseteq R_{* L}$. This case is dual to Case 2, and a dual argument shows that $C$ contains an element from $\mathcal{G}_{L}$ in this case.

Case 4: $C \nsubseteq R_{J *} \cup R_{* L}$. Then there exists an element $r \in C \backslash\left(R_{J *} \cup R_{* L}\right)$. It can be recorded in the form

$$
r=\sum_{i \in I, \lambda \in \Lambda} r_{i \lambda} e_{i \lambda}
$$

where all $r_{i \lambda} \in F$. By the choice of $r$, there exists $\mu \notin L$ and $j \notin J$ such that $r_{j \mu} \neq 0$. The definition of $L$ shows that $p_{\mu j^{\prime}} \neq 0$ for some $j^{\prime}$. Likewise, the definition of $J$ shows that $p_{\mu^{\prime} j} \neq 0$ for some $\mu^{\prime}$.

Consider the product $d=e_{j \mu^{\prime}} \cdot r \cdot e_{j^{\prime} \mu} \in C$. Since each row and every column of $P$ has at most one nonzero entry, we get $p_{\lambda j}=0$ for all $\lambda \neq \mu^{\prime}$, and $p_{\mu i}=0$ for all $i \neq j^{\prime}$. It follows that

$$
d=p_{\mu^{\prime} j} r_{j \mu} p_{\mu j^{\prime}} e_{j \mu} \in C .
$$

Since $\operatorname{wt}(d)=1$, we get $\operatorname{wt}(C)=1$. This contradiction completes the proof.

Proof of Theorem 1. Condition (i) follows directly from Theorem 2 and conditions (iv), (v) and (vi) of Proposition 3.

It is well known and easy to verify that a multiple clusterer with a cluster set of weight $W$ can correct $\left\lfloor\frac{W-1}{2}\right\rfloor$ errors of the individual clusterers. Therefore condition (ii) follows from (i).

\section{Applications and Open Question}

Our article makes the very first step in the investigation of the properties of Munn rings motivated by the information security applications. It contains only theoretical results of independent interest, which can be used to guide future experiments for determining how the constructions perform in practical situations. 
There do not exist exact and conclusive theoretical criteria of efficiency in clustering and data mining. This is also confirmed by the so-called "no free lunch" theorems in search, optimisation, and machine learning (see, for example, [42]). The efficiency of applications is always decided on the basis of experimental research. The results of experiments usually depend on particular application area and are evaluated using statistical methods (see, for example, [5]).

The present paper is motivated by the information security applications and aims to obtain results essential for providing guidance to the design of future experimental work. Theorems 1 and 2 characterize a class of multiple clusterers in the Munn rings, which are optimal with respect to property (A1). This result is essential, because in the design of experiments the researchers are mainly interested in the cluster sets satisfying the basic properties (A1), (A2), (A3) and (A4). However, the following problem motivated by property (A2) remains unresolved.

Problem 1 Let $F$ be a finite field, and let $R=M[F ; I, \Lambda ; P]$ be a Munn ring over $F$ with sandwichmatrix $P$. For every positive integer $m$, describe all cluster sets of the form $\operatorname{id}\left(g_{1}, \ldots, g_{k}\right)$ in $R$ with weight $m$ and the largest possible information rate.

A solution to this problem could help to choose cluster sets satisfying the basic properties (A1) and (A2) simultaneously for experimental evaluation in the future. Several separate experimental publications by a number of authors would be required for conclusive evaluation of the practical performance of applications like this in various areas. We refer to the monographs [41], [45] and recent articles [15, 19, $28,43,44]$ for examples of experimental investigations of this kind.

\section{Acknowledgements}

The first author was supported by Discovery grant DP0449469 from Australian Research Council. The second author was supported by Queen Elizabeth II Fellowship, Discovery grant DP0211866 and Linkage grant LP0990908 from Australian Research Council. The third author was supported by Linkage grant LP0776267 from Australian Research Council. The fourth author was supported by an RIBG grant of the University of Ballarat. The 1st, 2nd, 3rd, 5th and 6th authors were supported by a Ballarat-Deakin collaboration grant.

\section{References}

[1] Antony, N., Coleman, C. and D. Easdown, Group presentations for a class of radical rings of matrices, "Semigroups, Algorithms, Automata and Languages" (Coimbra, 2001), World Sci. Publ., River Edge, NJ, 2002, 293-311.

[2] Bagirov, A.M. and J.L. Yearwood, A new nonsmooth optimization algorithm for minimum sum-ofsquares clustering problems, European J. Operational Research 170 (2006), 578-596.

[3] Bagirov, A.M., Rubinov, A.M. and J.L. Yearwood, A global optimization approach to classification, Optim. Eng. 3 (2002), 129-155. 
[4] Bagirov, A.M., Rubinov, A.M., Soukhoroukova, N.V. and J.L. Yearwood, Unsupervised and supervised data classification via nonsmooth and global optimization, Top 11 (2003), 1-93.

[5] Boslaugh, S. and P. Watters, "Statistics in a Nutshell: A Desktop Quick Reference", O'Reilly \& Associates, 2008.

[6] Cárdenas, A.A. and J.S. Baras, Evaluation of classifiers: practical considerations for security applications, In: "Evaluation Methods for Machine Learning", The AAAI Press, Menlo Park, California, 2006, 30-35.

[7] Cazaran, J. and A.V. Kelarev, Generators and weights of polynomial codes, Arch. Math. (Basel) 69 (1997), 479-486.

[8] Cazaran, J., Kelarev, A.V., Quinn, S.J. and D. Vertigan, An algorithm for computing the minimum distances of extensions of $B C H$ codes embedded in semigroup rings, Semigroup Forum 73 (2006), $317-329$.

[9] Crabb, M.J., McGregor, C.M. and W.D. Munn, A property of the complex semigroup algebra of a free monoid, J. Aust. Math. Soc. 81 (2006)(1), 97-103.

[10] Easdown, D. and W.D. Munn, Trace functions on inverse semigroup algebras, Bull. Austral. Math. Soc. 52 (1995)(3), 359-372.

[11] Giacinto, G., Roli, F. and L. Didaci, Fusion of multiple classifiers for intrusion detection in computer networks, Pattern Recognition Letters 24(2003)(12), 1795-1803.

[12] Hall, T.E., The radical of the algebra of any finite semigroup over any field, J. Austral. Math. Soc. Ser. A 11 (1970), 350-352.

[13] Howie, J.M., "Fundamentals of Semigroup Theory", Clarendon Press, Oxford, 1995.

[14] Jackson, M. and M.V. Volkov, Undecidable problems for completely 0-simple semigroups, J. Pure Applied Algebra 213 (2009) (10), 1961-1978.

[15] Kang, B.H., Kelarev, A.V., Sale, A.H.J. and R.N. Williams, A new model for classifying DNA code inspired by neural networks and FSA, Lect. Notes Computer Science 4303 (2006), 187-198.

[16] Kelarev, A.V., "Ring Constructions and Applications", World Scientific, River Edge, NJ, 2002.

[17] Kelarev, A.V., "Graph Algebras and Automata", Marcel Dekker, New York, 2003.

[18] Kelarev, A.V., Göbel, R., Rangaswamy, K.M., Schultz, P. and C. Vinsonhaler, "Abelian Groups Rings and Modules", Contemporary Mathematics 273, American Mathematical Society, New York, 2001.

[19] Kelarev, A., Kang, B. and D. Steane, Clustering algorithms for ITS sequence data with alignment metrics, Lect. Notes Artificial Intelligence 4304 (2006), 1027-1031.

[20] Kelarev, A.V. and D.S. Passman, A description of incidence rings of group automata, Contemporary Mathematics 456(2008), 27-33. 
[21] Kelarev, A.V. and C.E. Praeger, On transitive Cayley graphs of groups and semigroups, European J. Combinatorics 24 (2003)(1), 59-72.

[22] Kelarev, A., Ryan, J. and J. Yearwood, Cayley graphs as classifiers for data mining: The influence of asymmetries, Discrete Mathematics 309 (2009)(17), 5360-5369.

[23] Kelarev, A.V. and O.V. Sokratova, On congruences of automata defined by directed graphs, Theoretical Computer Science 301 (2003), 31-43.

[24] Kelarev, A.V., Yearwood, J.L. and M.A. Mammadov, A formula for multiple classifiers in data mining based on Brandt semigroups, Semigroup Forum 78(2009)(2), 293-309.

[25] Kelarev, A.V., Yearwood, J.L. and P.W. Vamplew, A polynomial ring construction for classification of data, Bull. Aust. Math. Soc. 79 (2009), 213-225.

[26] Kelarev, A.V., Yearwood, J.L. and P. Watters, Rees matrix construction for clustering of data, J. Aust. Math. Soc. 87 (2009), 377-393

[27] López-Permouth, S.R., Shum, K.P. and N.V. Sanh, Kasch modules and pV-rings, Algebra Colloq. $12(2005)(2), 219-227$.

[28] McCombie, S., Watters, P., Ng, A. and B. Watson, Forensic characteristics of phishing - petty theft or organized crime?, Proc. 4th Internat. Conf. on Web Information Systems and Technologies, WEBIST (Madeira, Portugal, 2008).

[29] Munn, W.D., Some results on semigroup-graded rings, "Semigroups, algorithms, automata and languages" (Coimbra, 2001), World Sci. Publ., River Edge, NJ, 2002, 215-234.

[30] Munn, W.D. D-faithful semigroup-graded rings, Proc. Edinb. Math. Soc. 45 (2002)(3), 549-556.

[31] Munn, W.D. Rings graded by inverse semigroups, "Semigroups" (Braga, 1999), World Sci. Publ., River Edge, NJ, 2000, 136-145.

[32] Munn, W.D., Rings graded by bisimple inverse semigroups, Proc. Roy. Soc. Edinburgh Sect. A 130 (2000)(3), 603-609.

[33] Munn, W.D., A class of band-graded rings, J. London Math. Soc. 45 (1992)(1), 1-16.

[34] Munn, W.D., Nil right ideals in inverse semigroup algebras, Semigroup Forum 44 (1992)(1), 93-95.

[35] Munn, W.D., The Jacobson radical of a band ring, Math. Proc. Cambridge Philos. Soc. 105 (1989)(2), 277-283.

[36] Munn, W.D., Nil ideals in inverse semigroup algebras, J. London Math. Soc. 35 (1987)(3), 433-438.

[37] Munn, W.D., The algebra of a combinatorial inverse semigroup, J. London Math. Soc. 27 (1983)(1), $35-38$.

[38] Pellikaan, R., Wu, X.-W. and S. Bulygin, "Codes and Cryptology as Applications of Algebra, Combinatorics and Geometry", Cambridge University Press, 2010.

[39] Reilly, N.R., Varieties generated by completely 0-simple semigroups, J. Aust. Math. Soc. 84 (2008)(3), 375-403. 
[40] Silva, P.V., A note on primeness of semigroup rings, Proc. Roy. Soc. Edinburgh Sect. A 120 (1992)(34), 191-197.

[41] Witten, I.H. and E. Frank, "Data Mining: Practical Machine Learning Tools and Techniques", Elsevier/Morgan Kaufman, Amsterdam, 2005.

[42] Wolpert, D.H., The lack of a priori distinctions between learning algorithms, Neural Computation 8 (1996)(7), 1341-1390.

[43] Yearwood, J.L., Bagirov, A.M. and A.V. Kelarev, Optimization methods and the $k$-committees algorithm for clustering of sequence data, J. Applied Computational Math. 8 (2009)(1), 92-101.

[44] Yearwood, J.L., Kang, B.H. and A.V. Kelarev, Experimental investigation of classification algorithms for ITS dataset, PKAW 2008, Pacific Rim Knowledge Acquisition Workshop, part of PRICAI-08, Tenth Pacific Rim Internat. Conf. Artificial Intelligence, 262-272.

[45] Yearwood, J.L. and M.A. Mammadov, "Classification Technologies: Optimization Approaches to Short Text Categorization", Idea Group Inc., 2007. 